\title{
A novel biphasic scaffold supports meniscal tissue repair in ex vivo and in vivo models
}

\author{
Daniel Howard ${ }^{1}$, Edi Tanase ${ }^{1}$, John Wardale ${ }^{1}$, Frances Henson ${ }^{1,2}$ \\ ${ }^{1}$ Division of Trauma and Orthopaedic Surgery, Department of Surgery, University of Cambridge, Addenbrooke's Hospital, Hills' \\ Road, Cambridge, CB3 OES, UK \\ ${ }^{2}$ Department of Veterinary Medicine, University of Cambridge, Madingley Road, Cambridge, CB3 OES, UK
}

Correspondence: Frances Henson

E-mail: Fmdh1@cam.ac.uk

Received: July 26, 2016

Published online: October 12, 2016

\begin{abstract}
To cite this article: Daniel Howard, et al. A novel biphasic scaffold supports meniscal tissue repair in ex vivo and in vivo models. Musculoskelet Regen 2016; 2: e1411. doi: 10.14800/mr.1411.

Copyright: (c) 2016 The Authors. Licensed under a Creative Commons Attribution 4.0 International License which allows users including authors of articles to copy and redistribute the material in any medium or format, in addition to remix, transform, and build upon the material for any purpose, even commercially, as long as the author and original source are properly cited or credited.
\end{abstract}

\section{Introduction}

The meniscus is a fibrocartilagenous tissue that functions to stabilise the knee joint, to transmit load and to absorb shock. Meniscal injuries are common and the incidence of these injuries is increasing, particularly within an ageing population. In addition to the direct pain caused by meniscal damage and degeneration, mensical pathology leads to osteoarthritis (OA) of the knee.

Different tissue engineering strategies are currently being developed to treat damaged menisci including the use of decellularised allogeneic menisci ${ }^{[1]}$, synthetic scaffolds ${ }^{[2,3]}$ and biological scaffolds ${ }^{[4,5]}$. Whilst biological scaffolds are biocompatible and biodegradable, they often do not have the necessary biomechanical properties required to withstand the forces experienced by the meniscus. The meniscus requires high circumferential tensile strength to resist the compression and extrusion from the joint space that loading induces ${ }^{[6]}$. It has been shown that a biphasic collagen/synthetic polymer fibre scaffold mimics the tensile and hoop stress behaviour of normal meniscus under compressive loading ${ }^{[6]}$, indicating that a composite scaffold might provide the biomechanical strength needed. Our group has developed collagen fibres for a range of applications ${ }^{[7,8]}$, which can be incorporated into a collagen sponge to increase strength ${ }^{[9]}$. We have previously described the production of a novel collagen sponge reinforced with collagen fibres that can support meniscal cell growth in vitro ${ }^{[10]}$.

Whilst a scaffold will provide a structural support for the ingrowth of meniscal repair tissue, tissue engineering strategies have shown that incorporation of biological factors onto scaffolds can promote healing in the meniscus. Studies have shown that the addition of Platelet Rich Plasma (PRP) to scaffolds can enhance meniscal repair ${ }^{[11,12]}$. We have previously demonstrated that the combination of collagen scaffolds with PRP allows adsorption and extended release of biologically active growth factors and supports meniscal cell growth in vitro ${ }^{[10,13]}$.

The aims of this study were i) to investigate whether a novel biphasic collagen scaffold supports meniscal repair in an ovine meniscal defect model in vivo, ii) to investigate meniscal cell ingrowth and ECM production in an ex-vivo model and iii) to investigate the effect platelet rich plasma 




Figure 1. Photograph to show fibre orientation in the scaffold.

(PRP) on the response of meniscal elements to the scaffold.

\section{Materials and Methods}

Animals This study received approval from the UK Home Office and local research ethics committee. Whole ovine menisci from healthy stifle joints for the ex vivo models (EVM) were obtained from adult (aged $2-2.5$ years) female Welsh Mountain animals euthanized for non-orthopaedic reasons $(n=8)$. For the in vivo study, a total of 36 adult female Welsh mountain sheep were used in this study, $n=6$ per treatment group.

Scaffolds The scaffold used in this study was a collagen sponge with $300 \mu \mathrm{m}$ open pores, reinforced with bands of $150 \mu \mathrm{m}$ internal strengthening fibres ${ }^{[10]}$. The scaffold was constructed with strengthening fibres oriented so that they were the equivalent of parallel to the cranial surface of the meniscus (Fig. 1). Scaffolds were sterilised by gamma irradiation prior to use.

Production of PRP $6 \mathrm{ml}$ of sheep blood was collected into Acid Citrate Dextrose anticoagulant at a concentration of $1: 10$ and then centrifuged at $400 \mathrm{~g}$ for 10 minutes and plasma decanted to remove the red blood cells. The platelets were concentrated by centrifugation at $1000 \mathrm{~g}$ for 15 minutes. Platelets were resuspended in $400 \mu \mathrm{ls}$ of plasma. PRP cell composition profile was examined by fixing PRP in filtered, centrifuged and decanted ACDA: 4\% PFA: PBS, 1:1:1 preserving solution for flow cytometery using forward and side scatter for cell counts on a Beckman Coulter FC 500 with analysis on CXP Flowplot.

Surgical technique. The surgical technique was identical in each animal and performed under strict asepsis by a single surgeon. Surgery was performed under general anaesthesia. The left femoro-tibial joint was opened via a medial approach, leaving the medial collateral ligament intact. The superior surface of the medial meniscus was exposed and a 3 $\mathrm{mm}$ diameter circular defect was created using a modified biopsy punch. Once the defect was created it was either left unfilled ('Defect group' = Group D), the defect filled with a 3mm diameter scaffold plug ('Scaffold group' = Group S) or the defect filled with a scaffold plug pre-soaked with autologous PRP ('Scaffold + PRP group' = Group SP). Joints were sutured in a routine fashion and the animals allowed to fully weight bear post-operatively. The animals were housed in large indoor pens for 14 days post-surgery. Subsequently they were turned out into fields. Animals were euthanized with an overdose of anaesthetic at 8 and 24 weeks post-surgery.

Gait analysis Weight bearing on the operated limb was measured before and 2,5 and 8 weeks after surgery using an AMTI force plate (BP600600-1000) as described previously ${ }^{[10]}$. The weight bearing on the limb was quantified as $\mathrm{N} / \mathrm{kg}$ body weight for each individual animal and the data combined for each group to allow analysis.

Gross meniscal anatomy Digital images (600 dpi) of excised menisci were captured on a flatbed scanner. The percentage of the defect infilled with repair tissue at the surface was measured via image $\mathrm{J}$ software ${ }^{[14]}$.

Quantification of meniscal hardness. Meniscal tissue hardness (defined as a material's ability resistance to permanent indentation) was tested using a Shore S1 durometer that has been used previously to measure cartilage hardness ${ }^{[15]}$. A measurement was taken centrally at the defect site and $5 \mathrm{~mm}$ anterior, posterior to the defect centre and at the mid-point of the central region on the superior and inferior surfaces and on the superior and inferior surface of the medial menisci. A mean hardness score was acquired for each meniscus. As a control, the same points were measured on the unoperated medial meniscus of the other leg in the same animal, with all animals being evaluated individually. In the control animals the mean hardness score was allocated a value of $100 \%$ to represent the normal hardness of the tissue.

Histological examination of the menisci. The meniscus was excised and fixed with $4 \%$ paraformaldehyde and routinely embedded for wax histology. Sections of the menisci at the defect site were stained with Safranin O and counter stained with fast green and Gills II Haematoxylin. The histological appearance of the meniscus was scored using a scoring system described by Zhang ${ }^{[16]}$ (Table 1).

Antibodies The antibodies used for this study for Western blotting were rabbit polyclonal anti-type I collagen (Abcam, UK), rabbit monoclonal anti-versican (Abcam, UK), rabbit 
http://www.smartscitech.com/index.php/mr

Table 1 Histological scoring system

\begin{tabular}{|c|c|c|}
\hline Characteristic & Subcategory & Score \\
\hline \multirow[t]{5}{*}{ Nature of the predominant tissue } & Typical fibrocartilage & 5 \\
\hline & $>50 \%$ fibrocartilage & 4 \\
\hline & $\begin{array}{l}50 \% \text { fibrocartilage } / 50 \% \text { fibrous tissue or } \\
\text { scaffold }\end{array}$ & 3 \\
\hline & $>50 \%$ fibrous tissue & 2 \\
\hline & Fibrous tissue & $\mathbf{0}$ \\
\hline \multirow[t]{4}{*}{ Toluidine blue staining of matrix } & Normal staining & 3 \\
\hline & Moderate staining & 2 \\
\hline & Slight staining & 1 \\
\hline & None & $\mathbf{0}$ \\
\hline \multirow[t]{4}{*}{ Surface } & Smooth and intact & 3 \\
\hline & Superficial lamination & 2 \\
\hline & Slight disruption & 1 \\
\hline & Severe disruption & $\mathbf{0}$ \\
\hline \multirow[t]{3}{*}{ Integrity } & Normal structure & 2 \\
\hline & Slight disruption & 1 \\
\hline & Severe disruption & $\mathbf{0}$ \\
\hline \multirow[t]{3}{*}{ Thickness } & $75-100 \%$ of adjacent tissue & 2 \\
\hline & $50-74 \%$ of adjacent tissue & 1 \\
\hline & $0-49 \%$ of adjacent tissue & $\mathbf{0}$ \\
\hline \multirow[t]{3}{*}{ Bonding to host tissue } & Bonded & 2 \\
\hline & Partially bonded & 1 \\
\hline & Not bonded & $\mathbf{0}$ \\
\hline \multirow[t]{4}{*}{ Repair tissue in the defect } & Normal cell morpholgy and matrix staining & 3 \\
\hline & Cell clustering and normal matrix staining & 2 \\
\hline & Decreased matrix staining & 1 \\
\hline & Poor or no matrix staining & $\mathbf{0}$ \\
\hline \multirow[t]{4}{*}{ Overall healthy cartilage cell number } & Similar to normal fibrocartilage & 3 \\
\hline & Slightly less than normal fibrocartilage & 2 \\
\hline & Much less than normal fibrocartilage & 1 \\
\hline & Acellular & $\mathbf{0}$ \\
\hline \multirow[t]{5}{*}{ Matrix porosity } & Thick matrix no pores & 5 \\
\hline & Thick matrix some pores & 4 \\
\hline & Semithick matrix with many pores & 3 \\
\hline & Porous matrix & 2 \\
\hline & Fibrous matrix & $\mathbf{0}$ \\
\hline Maximum possible score & & 31 \\
\hline
\end{tabular}
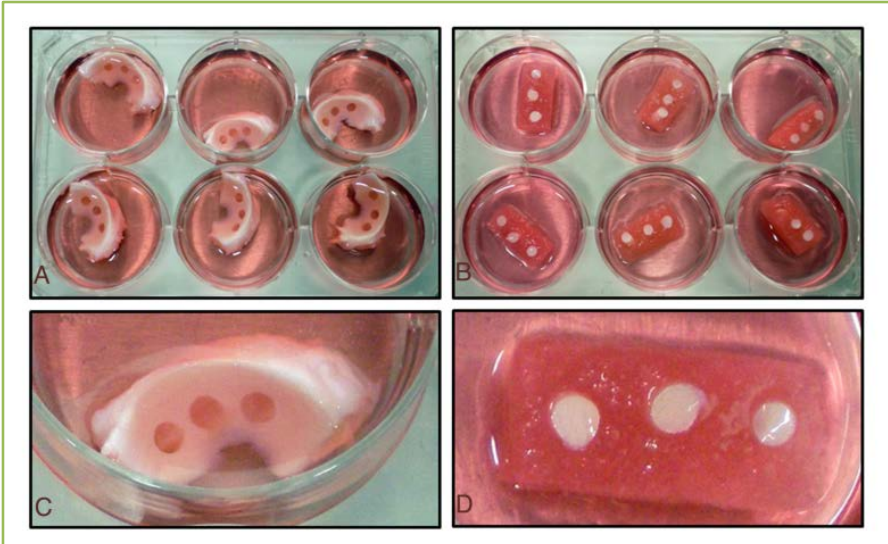

Figure 2. Photographs to show construction of ex vivo models (EVM). A and C Internal EVMs in which a $3 \mathrm{~mm}$ diameter plug of scaffold has been inserted into a $3 \mathrm{~mm}$ diameter hole in an adult ovine meniscus. A Examples of constructs, $\mathrm{C}$ a close up picture of one construct. B and D External EVM in which in which $3 \mathrm{~mm}$ diameter plugs of meniscus (derived from making the holes seen in $A$ and $C$ ) have been inserted into $3 \mathrm{~mm}$ diameter holes in the scaffold. B Examples of constructs, D a close up picture of one construct.

monoclonal anti-lumican (Abcam, UK), rabbit monoclonal vimentin (Abcam, UK), (rabbit polyclonal anti-smooth muscle actin (SMA) (Abcam, UK) and rabbit polyclonal anti-GAPDH (Sigma, UK). The antibodies used for this study for Immunohistochemistry (IHC) were rabbit polyclonal monoclonal anti-mouse types I and II Collagen (MP Biomedicals, US) and monoclonal anti-vimentin (Dylight 488 conjugated (Abcam, UK).

Immunohistochemical examination Standard IHC techniques were used as previously described ${ }^{[10]}$. Type I and II collagen antibodies (1:200 dilution) were used with a biotinyated secondary anti-mouse antibody (Sigma, UK) and developed with diaminobenzamine (DAB) to localise types I and II collagen. Vimentin (1:200 dilution) was directly visualised under fluorescent microscopy.

Ex vivo model (EVM) Preparation Isolated meniscus were incubated in culture medium (DMEM containing 10\% foetal calf serum, 100iu/100ug/ml penicillin/streptomycin, 10ug/ml Gentamycin, 2.5ug/ml Amphotericin B and 20ug/ml ascorbate-2-phosphate (Sigma)) for 24hrs prior to exerimental manipulation. Two types of EVM were created; 1. Internal where a scaffold is surrounded with tissue to directly mimic the in vivo experiments and 2. External where an explant is surrounded by scaffold (Fig. 2). Internal EVMs 


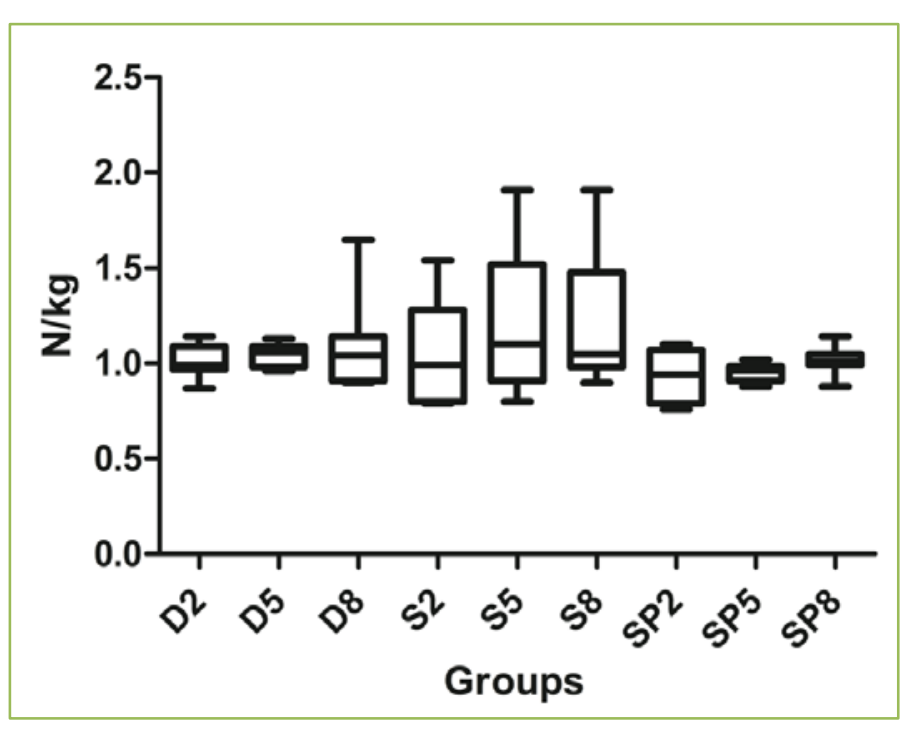

Figure 3. Weight bearing on the operated leg pre surgery, 2, 5 and 8 weeks post surgery in the three groups of animals described in this study normalised to the pre-surgery weight bearing. Measurements of weight bearing at $2 \mathrm{w}$ post-surgery did not find any significant differences between groups or compared to pre surgical values. Subsequent measurements of weight bearing did not find any significant differences between groups or time points. These results indicate that the surgical procedure was mild and did not cause a significant lameness post-surgery. There was not alteration in the weight bearing of operated legs between groups. D defect only, S - defect + scaffold, SP - defects + scaffold + PRP.

were created by removing full depth $3 \mathrm{~mm}$ diameter meniscal cartilage explants from normal meniscal tissue using a biopsy punch (Kai Medical). Scaffold discs (3mm diameter and $4 \mathrm{~mm}$ depth) were soaked in culture media or PRP for 5 minutes and inserted into the holes in the meniscus. External EVMs were constructed by using the $3 \mathrm{~mm}$ diameter explants derived during the construction of the IEVMs and placing them into $3 \mathrm{~mm}$ diameter holes made in scaffold material. EVM were cultured in 12 well plates containing complete media for up to 28 days.

Cell Culture Isolated meniscal cells were obtained so that a population of 'native' meniscal cells were available for protein analysis. Mensical cells were prepared by finely chopping fresh ovine meniscal cartilage and digesting overnight at $37^{\circ} \mathrm{C}$ with shaking in $0.2 \%$ collagenase A (Sigma). Cells were washed and plated out in culture medium as above and first passage cells were extracted for western blotting as detailed below.

Western Blotting At EVM harvest, scaffolds and tissue were separated. Proteins were extracted from scaffolds and cartilage in cell extraction buffer (Life Technologies) containing a proteinase inhibitor cocktail before running on SDS electrophoresis and Western blotting as previously described $^{[17]}$.

Histology At 3, 10, 17 and 24 days in culture, EVMs were fixed in $4 \%$ paraformaldehyde and snap-frozen. Sections $(8 \mu \mathrm{m}$ thick) were obtained and stained with Vectashield (Vector Laboratories) containing 4',6-diamidino-2-phenylindole (DAPI) to stain the cell nuclei. Images were acquired and the distance that the meniscal cells had migrated in to the scaffold calculated using ImageJ software. The mean value of 3 different EVM was calculated.

Immunhistochemistry After 24 days EVMs were harvested for immunohistochemistry. Frozen sections $(8 \mu \mathrm{m}$ thick) were fixed using a 1:1 solution of acetone and methanol before probing with primary antibodies and FITC-conjugated secondary antibodies and mounting in Vectashield (Vector Laboratories) containing DAPI.

\section{Results}

\section{In vivo model}

PRP analysis The PRP preparations contained $1.37 \times 10^{6}$ $\left(+/-4.6 \times 10^{4}\right)$ platelets per microlitre compared to whole blood samples which contained $1.8 \times 10^{5}$ (+/- $1.1 \times 10^{5}$ ) platelets per microlitre.

Gait analysis The weight bearing on the operated limb was measured before surgery, and 2, 5 and 8 weeks post surgery and expressed as N/kg. Pre-surgery, there was no difference between the experimental groups, with an average $\mathrm{N} / \mathrm{kg}$ of 5.6 of weight born on the leg that was subsequently operated on. Measurements of weight bearing at $2 \mathrm{w}$ post-surgery did not find any significant differences between groups or compared to pre surgical values. Subsequent measurements of weight bearing did not find any significant differences between groups or time points (Fig. 3).

Gross morphology and tissue hardness. At 8 and 26 weeks post-surgery joints were recovered and opened. There was no evidence of increased synovial fluid or synovitis in the joint. Mensical defects were still present in operated menisci (Fig. 4). The defects were seen as disruptions of the meniscal surface with new tissue infilling the defect regions in all groups. At $8 \mathrm{w}$ there was no difference in percentage surface infill between groups, however, at $26 \mathrm{w}$ there was a significant increase in surface infill in Group SP compared to Group S (Fig 4).

Tissue hardness was measured by Durometer indentation testing at 8 and 26 weeks post-surgery (Fig. 5). The average 


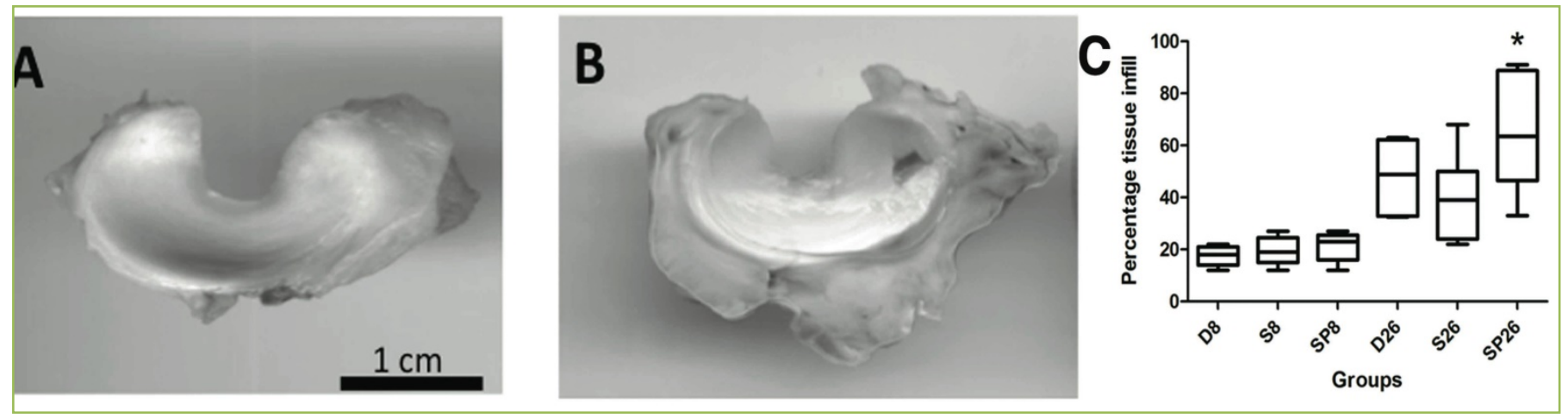

Figure 4. Photographs of menisci recovered from animals 8 w post surgery. A control meniscus from unoperated leg to show normal appearance of the ovine meniscus. B meniscus from animal in which only a defect was made in the meniscus (Group D), C meniscus from animal in which the defect in the meniscus was treated with a scaffold (Group S), D meniscus from animal in which the defect in the meniscus was treated with a scaffold plus PRP (Group SP). These menisci are the median scoring sample (histological scoring) from each of their respective treatment groups. In each meniscus the operated site is indicated with a black line. $3 b$. Graph to show the percentage tissue infill at the defects at $8 \mathrm{w}$ and $26 \mathrm{w}$ post surgery. There is a statistically significant $(\mathrm{p}<0.05)$ difference between Group $\mathrm{S}$ and Group $\mathrm{SP}$ in the $26 \mathrm{w}$ operated animals, indicating that the addition of PRP to the scaffold has enhanced the amount of surface tissue infill in this model. D - defect only, S - defect + scaffold, SP - defects + scaffold + PRP.



Figure 5. Graph to show the hardness of the repair tissue in the meniscus at $8 w$ and $26 w$ post-surgery. At 8 weeks post-surgery, there was a significant difference in the hardness of all 3 operated groups compared to the unoperated, control meniscus, however, there was no difference between the groups. At 26 weeks post-surgery the tissue hardness had increased compared to the hardness measured at 8 weeks post-surgery, however, again, there was no difference between the groups and no statistical difference between the groups and the unoperated, control meniscus.

hardness measurement for the control (unoperated, contralateral limb) meniscus was $59.1+/-6$ at 8 weeks post-surgery and $63.8+\backslash-7.2$ at 26 weeks post-surgery. At 8 weeks post-surgery, there was a significant difference in the hardness of all 3 operated groups compared to the unoperated, control meniscus, however, there was no difference between the groups. At 26 weeks post-surgery the tissue hardness had increased compared to the hardness measured at 8 weeks post-surgery, however, again, there was no difference between the groups and no statistical difference between the groups and the unoperated, control meniscus (Fig. 5).

Histological assessment of meniscal repair Subjectively, there was good infill of tissue into scaffolds at both 8 and 


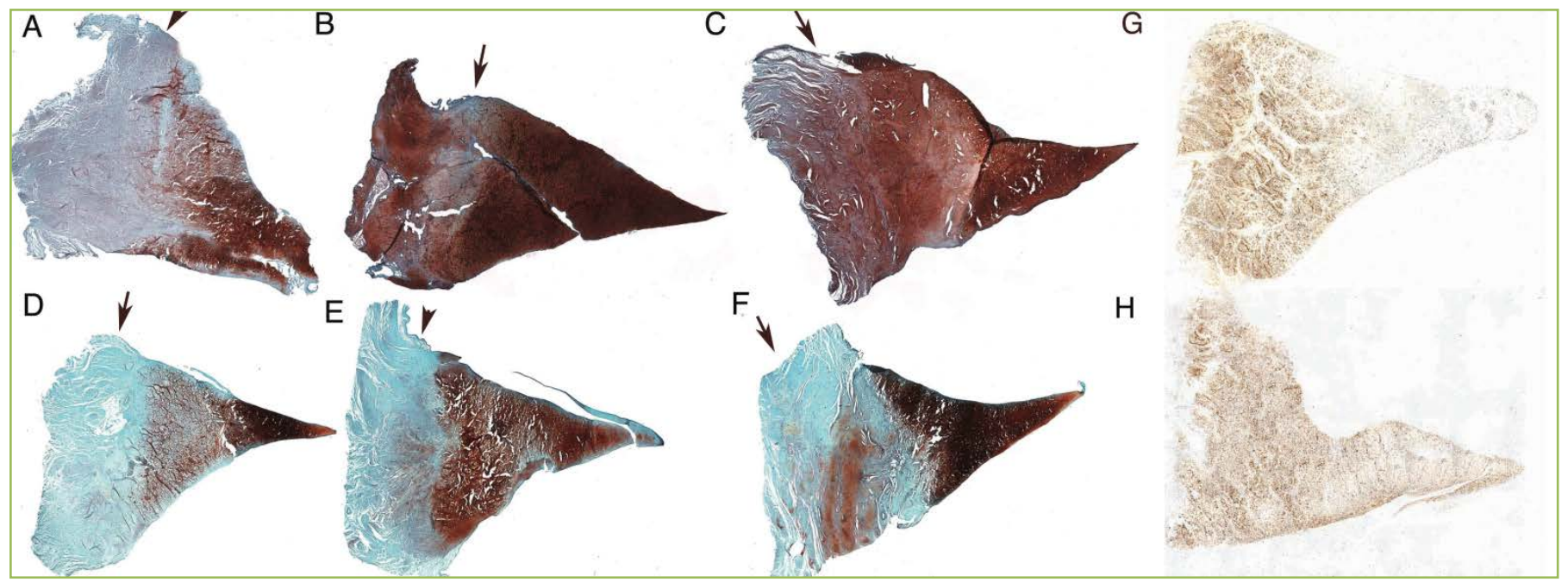

Figure 6. Histology and Immunohistochemistry of meniscal tissues. A-F Safranin $O$ stained whole menisci. A, B and $C$ retrieved $8 w$ post surgery, D, E and F retrieved 26w post surgery. A and D Group D (Defect), B and E Group S (Scaffold placed in defect), C and F Group SP (Scaffold plus PRP placed in defect). There is tissue infill in each of these menisci. The original defect is indicated with a line. In the $26 \mathrm{w}$ post surgery group there is more maturation of the extracellular matrix. In each section the black arrow indicates the position and orientation of the defect and each section represents the median section identified following quantitative scoring of healing. $G$ Representative example of type I collagen staining in repairing meniscus, $\mathrm{H}$ Representative example of versican staining in repairing meniscus. In each section the white arrow indicates the position and orientation of the defect.

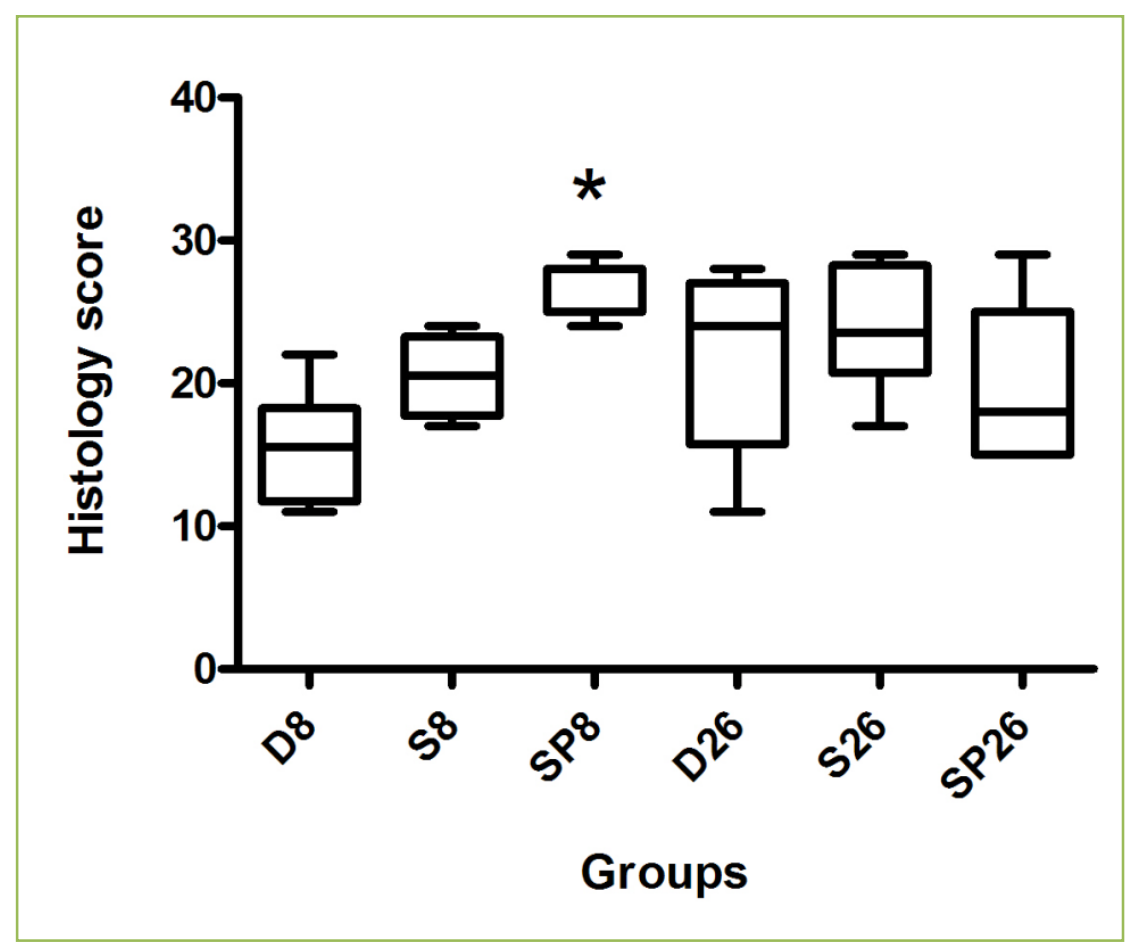

Figure 7. Quantitative scoring of repair of meniscal defects. A $8 w$ post surgery, B 26 weeks post surgery. At 8w post surgery there is a significant increase in histology score in the scaffold plus PRP group compared to the control and scaffold only group. At $26 \mathrm{w}$ post surgery there is no difference between groups.

26w (Fig. 6). The repair tissue stained extensively with Safranin O suggesting the presence of proteoglycan within the defect site. Defects were scored to provide quantitative data on the degree of healing. In the histological sections at $8 \mathrm{w}$ it was seen that the addition of PRP to the scaffold
(Group SP) significantly increased the healing (Fig. 7) compared to Groups D and S. By 26w there was no difference between experimental groups (Fig. 7). Remnants of scaffold material was detected in all Group S and SP at 8w but not at 26w post-surgery. 

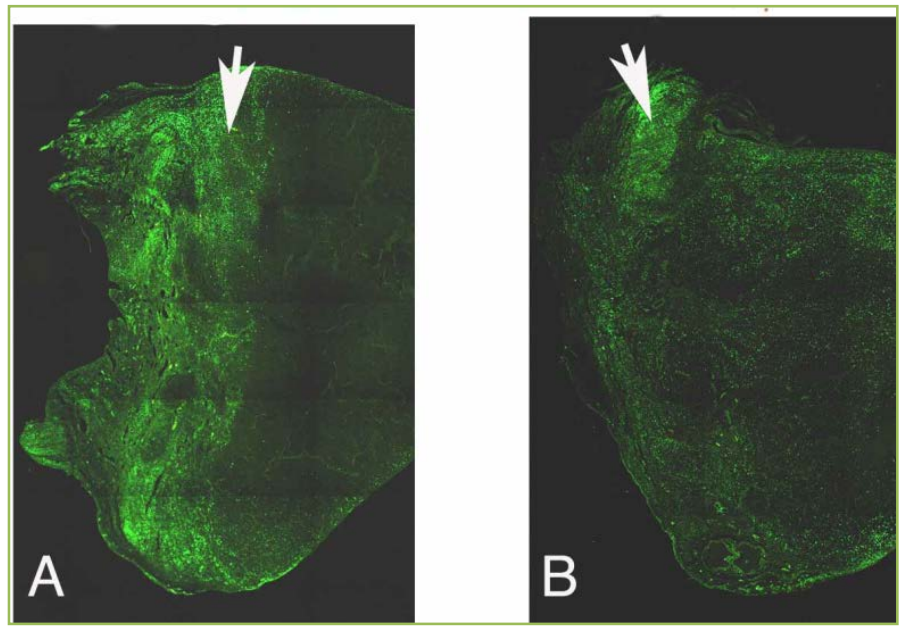

Figure 8. Vimentin immunohistochemistry (IHC) in repairing meniscus. in the meniscus. A $8 \mathrm{w}$ post surgery, B $26 \mathrm{w}$ post surgery. In $A$ there is an increase in the green fluorescent signal indicating the presence of vimentin at the edge of the meniscus adjacent to and extending through the repair tissue. In both sections the white arrow indicates the position and orientation of the defect.

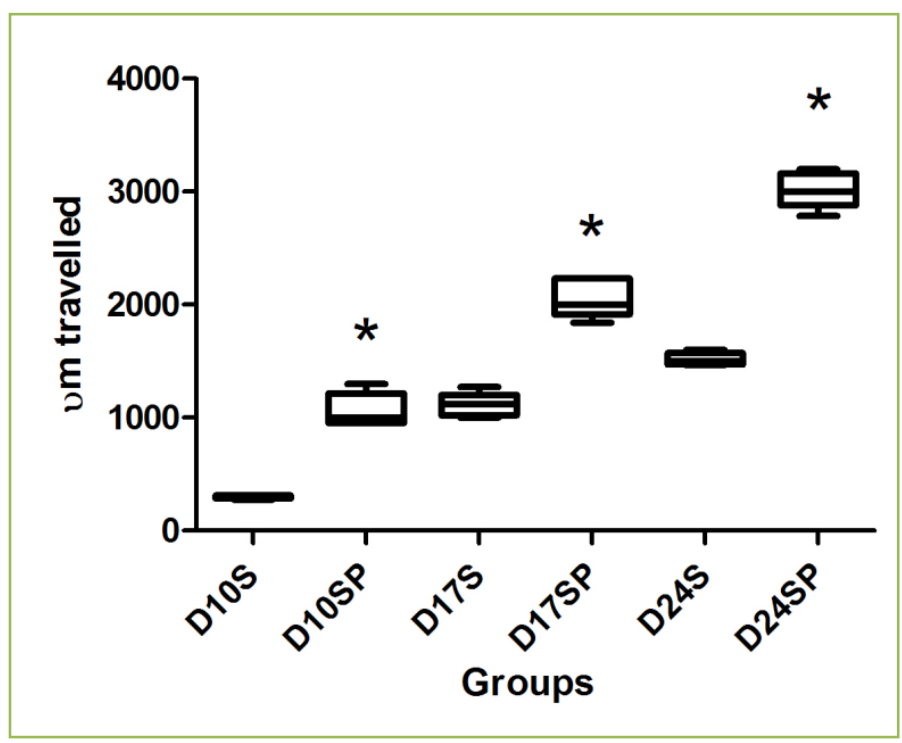

Figure 9. Graph to show quantification of distance that cells had migrated into scaffolds in ex vivo models. $D=$ day, $S-=$ scaffold alone, S+ scaffold plus meniscus, SP+ scaffold plus PRP plus meniscus. In Group S EVM, cells had migrated with a mean distance of $1500 \mu \mathrm{m}(+1-175)$ into the scaffold, whereas in the presence of PRP (Group SP) cells had migrated $2950 \mu \mathrm{m}(+\mid-297)$ into the scaffold.

Immunohistochemical assessment of meniscal tissue IHC of meniscal ECM markers demonstrated that collagen I and versican were present in the meniscus and within the healing defect at $8 \mathrm{w}$ and 26w post-surgery (Fig.6). An increase in vimentin positive cells was detected in the regions of the defects of samples at $8 \mathrm{w}$, this difference was not apparent at 26w (Fig. 8).

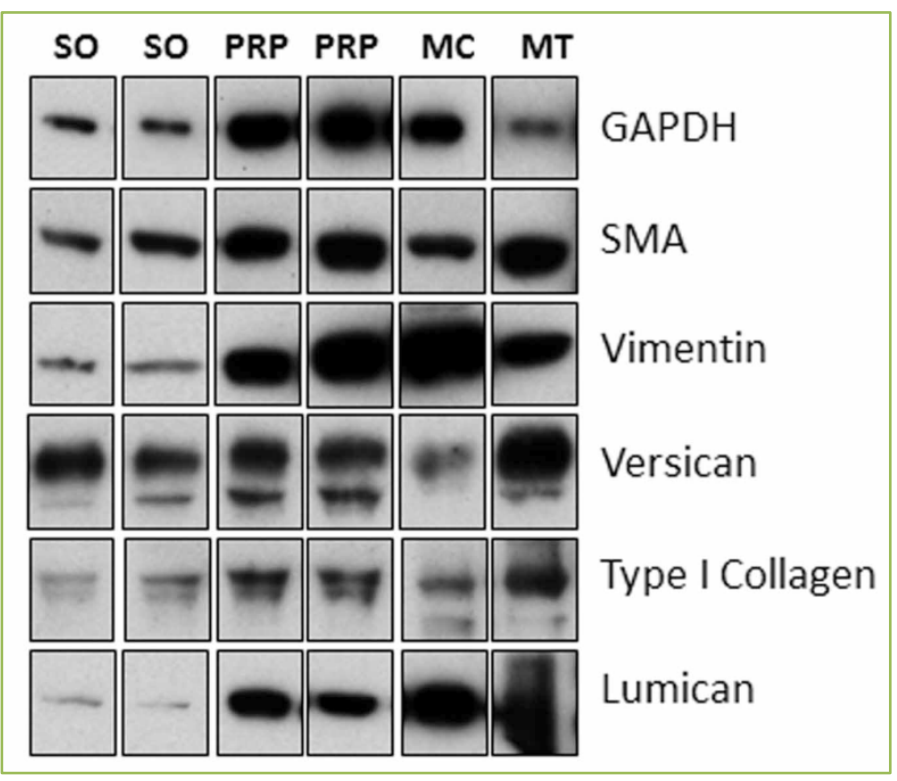

Figure 10. Western blots of meniscal tissue, meniscal cells, and scaffolds removed from external ex vivo models (two different samples). Each sample was Western blotted for smooth muscle actin (SMA), vimentin, versican, type I collagen, lumican. Cells obtained from within the scaffold produced collagen type I and versican, as is found in mature meniscus tissue. The production of luminca, also present in mature meniscal tissue was low in cells within the scaffold but increased in the presence of platelet rich plasma $(\mathrm{PRP}) . \mathrm{SO}=$ Scaffold only, $\mathrm{PRP}=$ Scaffold $+\mathrm{PRP}, \mathrm{MC}=$ Meniscal calls, MT = Meniscal tissue.

\section{Ex vivo models}

Histology In histological sections, cells were visible both around the cut edges of EVM and within the scaffolds at all time points studied. The distance that the cells had migrated into the scaffold was calculated. In the presence of PRP cells had migrated further at all time points studied compared to scaffold alone EVM. In plain EVM, cells had migrated with a mean distance of $1500 \mu \mathrm{m}(+\backslash-175)$ into the scaffold, whereas in the presence of PRP cells had migrated $2950 \mu \mathrm{m}$ $(+\backslash-297)$ into the scaffold by 24 days. (Fig. 9).

Western Blotting Western blots of extracts of meniscal tissues and cells that had been enzymatically isolated showed that they contained collagen type I and versican which are markers of meniscal ECM. Cells extracted from within internal and external EVMs also demonstrated the presence of these markers (Fig. 9). In addition to identifying markers of meniscal ECM proteins in the extracts from the scaffolds, Western blotting also identified SMA, lumican and vimentin within the meniscal tissue, cells and in the scaffolds (Fig. 10).

Analysis of the Western blots using densitometry demonstrated that the addition of PRP to the scaffold 




Figure 11. Western Blot densitometry of A. Internal ex vivo models (EVM) B External EVM (Internal). Type I collagen and versican, markers of meniscal extracellular matrix are detected in both types of constructs. Markers of migrating cells (smooth muscle actin (SMA) and vimentin) are increased in the presence of platelet rich plasma (PRP), significantly so in the external EVM. * denotes statistically significant difference $\mathrm{p}<0.05$.

enhanced SMA, lumican and vimentin in the scaffolds (Fig 11), with most effects being detected in the external EVM. Statistically significant data was achieved for the markers of cell migration/repair and for the overall cell number (GAPDH) in the external EVM.

\section{Discussion}

In this study we have demonstrated that a novel biphasic collagen scaffold can support meniscal defect repair in an in vivo model and support the ingrowth of meniscal cells in an ex vivo model. In addition we have demonstrated that the addition of PRP significantly enhances mensical cell migration and ECM production in vitro and promotes rapid defect healing in vivo.

In order to investigate the biocompatibility of the biphasic scaffold, scaffolds were implanted into a circular meniscal defect in an ovine model. At 8 and 26w post-surgery macroscopically there was comparable tissue infill into the repair site in all groups, demonstrating that the scaffold was able to support tissue healing. Quantitative analysis of the repair tissue showed that, at $8 \mathrm{w}$, there was a significant increase in the histology score in the defects treated with scaffolds including PRP compared to control (empty) defects and scaffolds alone, by 26w no differences were noted. At 26w the mean histology score for all animals was similar to the mean histology score of the scaffold + PRP defects at $8 \mathrm{w}$, indicating that the addition of PRP to the scaffolds had accelerated the healing more rapidly towards the maximum that could be achieved by $26 \mathrm{w}$. These results, i.e. some improvement in histology early in the repair process but no significant overall in vivo improvement at $26 \mathrm{w}$, illustrate the difficulties in interpreting results from experiments comparing treatments with and without augmentation of PRP. Recent clinical studies have shown both benefit ${ }^{[18]}$ and no benefit ${ }^{[19]}$ of augmenting meniscal repair with PRP in patients, while animal models have yielded similarly opposing results ${ }^{[12,20]}$.

The optimal pre-clinical model for the in vivo evaluation of meniscal scaffolds is not defined ${ }^{[21,22]}$. Ovine models have previously been used to evaluate meniscal repair and regenerative strategies ${ }^{[23,24]}$ and the structural properties and physiology of the ovine meniscus are relatively similar to that of the human meniscus ${ }^{[22,25]}$. It has been reported that the ovine meniscus has superior healing capacity compared to human meniscus, with tissue ingrowth seen in empty defect groups in other studies ${ }^{[21]}$. In this study the $8 \mathrm{w}$ post-surgery results were sufficiently different between groups such that any intrinsic healing capacity of ovine meniscus did not compromise the evaluation of the scaffold. However, by 26w post-surgery there were no difference between experimental groups, which could be partly related to the efficient intrinsic healing of the ovine meniscus.

In this experiment we also used measurements of weight bearing in the operated limb to indirectly measure joint pain post-surgery. We have previously shown that weight bearing measurements can significantly differentiate between treatment groups post-surgery in sheep ${ }^{15}$. In this study there were no differences detected in weight bearing pre- and post surgery, nor between treatment groups, indicating that this surgical procedure and treatments did not cause detectable discomfort either immediately or for up to 8 weeks post-surgery. The addition of PRP to joints can cause inflammation. In one study multiple intra-articular PRP injections PRP lead to moderate/severe pain in $67 \%$ of 
patients. In this study no differences were detected between groups, indicating that the inclusion of the scaffold +/- PRP was biocompatible and did not cause joint pain.

In this study, a full thickness cylindrical meniscal defect was used to evaluate scaffold performance. This defect type has been previously described in small animal models ${ }^{[11,26]}$ and is optimal to ensure that the scaffold remains in situ during the experimental period. Previous studies using partial meniscectomy models have reported scaffold loss as a significant impact on the analysis of results ${ }^{[4]}$. In this study we could detect the remnants of the scaffold in all animals at $8 \mathrm{w}$, indicating that the scaffold was retained in the defects and that this defect shape is an acceptable method of scaffold evaluation.

The finding that the biphasic scaffold could support tissue repair and that this repair was apparently accelerated by PRP has identified a potential use of this scaffold in meniscal defect repair in the clinic. However, whilst the in vivo data provides valuable information on the safety and biocompatibility of the scaffold, it does not permit evaluation of any mechanisms underlying meniscal repair. In order to investigate mechanisms and to begin to understand the role of PRP in potentiating meniscal healing we constructed cartilage/scaffold EVM.

The construction of EVMs for investigating regeneration therapies has been previously described for articular cartilage by our group ${ }^{[27]}$. EVM have several advantages over purely in vitro systems (e.g. cells seeded into scaffolds) in that the scaffold and any additives are in direct contact with the relevant damaged tissue and the architecture of the tissue supporting the cells is retained ${ }^{[28]}$. Our study has demonstrated that, when meniscal cartilage is co-cultured in contact with the biphasic scaffold, there is extensive migration of meniscal cells from the tissue into the scaffolds where they produce a meniscus appropriate ECM.

It is generally accepted that endogenous tissue repair is driven by progenitor cells located within the tissue that migrate to injury sites, proliferate and deposit new matrix ${ }^{[29]}$. In meniscus, these mobile cells have been termed meniscal fibrochondrocytes (MFC) and have been previously shown to have a significant capacity for migration in vitro ${ }^{[30,31]}$. There are, however, differences in cells isolated by enzymatic digestion and those grown out of meniscal explants. In the studies described here, the MFCs that entered the scaffolds are an outgrowth population, which have been shown to have progenitor properties in previous studies ${ }^{[32]}$. Further studies are underway to fully characterise the cell population mobilised to migrate from meniscus to scaffold in these experiments.
Following damage to the meniscus, signals initiate and support MFC migration. It is likely that it is a combination of mechanical (e.g. the release of cells from a dense ECM ${ }^{[32]}$ ) and biological factors drive the recruitment of cells into the scaffold. It has been shown that MFCs are mobilised by signals found in fibrin clots ${ }^{[33]}$ and that platelet derived growth factor (PDGF) and endothelin-1 promote migration of meniscal cells in vitro ${ }^{[30]}$. In this study we have demonstrated that the addition of PRP significantly enhanced the migration of the MFC into the scaffolds and increased the deposition of ECM within the scaffolds, as has been reported in other studies ${ }^{[12,34]}$. PRP contains both fibrin and a number of growth factors, including PDGF, any of which could be acting alone or in synergy to enhance MFC migration. Although PRP appeared to have a slight beneficial effect on extracellular matrix deposition, the data was not significant in either model. This implies that the initial effects of PRP in these co-culture models is on cell migration primarily rather than extracellular matrix formation.

Whilst the migratory capacity of MFC has been previously described, little has been reported on the mechanisms by which the migration is occurring. In this study we have shown that three markers reported to be associated with repair and cell migration in connective tissue cells; vimentin, lumican and SMA, are expressed by the MFC captured within the scaffold. Vimentin, is upregulated following injury to tissues, exerting a pleiotropic and context-dependent control on cells ${ }^{[35]}$. SMA, a contractile actin isoform, has a role in contractile activity within cells ${ }^{[36]}$ , and has been described in articular chondrocytes and meniscus ${ }^{[37,38]}$. SMA in damaged articular cartilage ${ }^{[39]}$ has been suggested to be a marker of the appearance of chondrocytes with repair capacity - "myochondrocytes" [39]. Lumican, a small, leucine-rich proteoglycan (SLRP) has been shown to play a role in tissue healing and cell migration [40-42].

No association of vimentin, SMA or lumican with healing has been previously reported in the meniscus. In this study we demonstrate the presence of vimentin, SMA and lumican in meniscal tissue, mensical cells and MFC captured into scaffolds. We also demonstrate that PRP upregulates these markers in MFC captured within the scaffold, with significant increases occurring in the external EVM co-cultures compared to the internal EVM. There are no previous reports of the effect of PRP on these proteins in meniscal tissues or MFC although PRP has been shown to promote the migration of a number of other cell types ${ }^{[43,44]}$ and PRP has been shown to increase SMA associated with a differentiation into myofibroblasts and the promotion of wound contraction ${ }^{[45]}$. Our observed effects of PRP may provide insight into the mechanism by which PRP promotes 
tissue repair and warrants further investigation. It is notable that vimentin, SMA and lumican were only increased significantly by PRP in the external EVM. It is possible that this is due to the cells in external EVM being relatively unconfined when they enter the scaffold compared to the space constraints of the internal EVM where only a small scaffold surface is offered. In this smaller scaffold space MFCs may experience contact inhibition between cells, which does not occur in the larger scaffold space offered by external EVM. Such 'contact inhibition of locomotion' ${ }^{[46]}$ could explain the reduction in migratory capacity in response to stimulation by PRP detected in MFC in the reduced scaffold surface space.

In conclusion, we have developed a novel biphasic collagen scaffold and shown that it is able to support meniscal repair in a defect model in vivo and to support meniscal cell ingrowth and ECM production in vitro. The addition of PRP to the scaffold enhanced healing at $2 \mathrm{~m}$ in vivo and significantly enhanced MFC migration in vitro, possibly through the intermediary of vimentin, SMA and lumican. Work is currently ongoing to characterise the MFC populations recruited into the scaffolds and to identify mechanisms underlying their mobilisation.

\section{Conflicting interests}

The authors have declared that no conflict of interests exist.

\section{Acknowledgements}

This study was funded by the NIHR project number II-3B-0109-10038.

\section{Author contributions}

DH was involved in the experimental design, acquired data, analysed data and contributed to the writing of the manuscript. ET acquired data, analysed data and contributed to the writing of the manuscript. JW was involved in the experimental design, acquired data, analysed data and contributed to the writing of the manuscript. FH was involved in the experimental design, acquired, analysed data and contributed to the writing of the manuscript. All authors have read and approved the final submitted manuscript.

\section{References}

1. Myers P, Tudor F. Meniscal allograft transplantation: how should we be doing it now? A systematic review. Arthroscopy 2015; 31: 911-925.

2. Bouyarmane H, Beaufils P, Pujol, Nakajima M, Bellemans J, Roberts S, et al. Polyurethane scaffold in lateral meniscus segmental defects: clinical outcomes at 24 months follow-up. Orthop Traumatol Surg Res 2014; 100: 153-157.

3. Verdonk P, Beaufils P, Bellemans J, Dijan P, Heinrichs E L, Huysse W, et al. Successful treatment of painful irreperable partial meniscal defects with a polyurethane scaffold:two year safety and clinical outcomes. Am J Sports Med 2012; 40: 844-853.

4. Gruchenberg K, Ignatius A, Friemert B, von Lübken F, Skaer N, Gellynck $\mathrm{K}$, et al. In vivo performance of a novel silk fibroin scaffold for partial meniscal replacement in a sheep model. Knee Surg Sports Traumatol Arthrosc 2015; 23: 2218-2229.

5. Linke R D, Ulmer M, Imhoff A B. Replacement of the meniscus with a collagen implant (CMI). Oper Orthop Traumatol 2006; 18 : 453-462.

6. Balint E, Gatt C, Dunn M G. Design and mechanical evaluation of a novel fiber-reinforced scaffold for mensicus repalcement. J Biomed Mater Res A. 2012; 100: 195-292.

7. Kew S J, Gwynne J H, Enea D, Brooks R, Rushton N, M B S, et al. Synthetic collagen fascicles for the regeneration of tendon tissues. Acta Biomater 2012; 8: 3723-3731.

8. Enea D, Gwynne J H, Kew S J, Arumagam M, Shepherd J, Brooks $\mathrm{R}$, et al. Collagen fibre implant for tendon and ligament biological augmentation. In vivo study in an ovine model. Knee Surg Sports Traumatol Arthrosc 2013; 21: 1783-1793.

9. Shepherd J H, Ghose S, Kew S J, Moavenian A, Best S M, Cameron R. Effect of fiber cross-linking on collagen-fibre reinforced collagen-chondroitin-6-sulfate materials for regenerating load-bearing soft tissues. J Biomed Mater Res A. 2013; 101: 176-184.

10. Howard D, Shepherd J, Kew S J, Hernandez P, Ghose S, Wardale $\mathrm{J}$, et al. Release of growth factors from a reinforced collagen GAG matrix supplemented with platelet rich plasma: Influence on cultured human meniscal cells. J Orthop Res 2014; 32: 273-278.

11. Ishida K, Kuroda R, Miwa M, Tabata Y, Hokugo A, Kawamoto T, et al. The regenerative effects of platelet-rich plasma on meniscal cells in vitro and its in vivo application with biodegradable gelatin hydrogel. Tissue Eng 2007; 13: 1103-1112.

12. Kwak H S, Nam J, Lee J H, Kim H J, Yoo J J Meniscal repair in vivo using human chondrocyte-seeded PLGA mesh scaffold pretreated with platelet-rich plasma. J Tissue Eng Regen Med, 2104.

13. Getgood A, Henson F M D, Brooks R, Fortier L A, Rushton N. Platelet-rich plasma activation in combination with biphasic osteochondral scaffolds - conditions for maximal growth factor production. Knee Surg Sports Traumatol Arthrosc 2011; 19: 1942-1947.

14. Schneider C A, Rasband W S, Eliceri K. NI Image to ImageJ: 25 years of image analysis. Nat Methods 2012; 9: 671-675.

15. Power J, Hernandez P, Guehring H, Getgood A, Henson F M D. Intra-articular injection of rhFGF-18 improves the healing in microfracture treated chondral defects in an ovine model. J Orthop Res 2014; 32: 669-676.

16. Zhang H, Leng $\mathrm{P}$, Zheng J. Enhanced meniscal repair by overexpression of hlGF-1 in a full thickness model. Clin Orthop Relat Res 2009; 467: 3165-3174.

17. Hernandez P, Whitty C, Wardale J, Henson F M D. New insights into the location and form of sclerostin. Biochem Biophys Res 
http://www.smartscitech.com/index.php/mr

Commun 2014; 446: 1108-1113.

18. Pujol N, Salle De Chou E, Boisrenoult P, Beaufils P. Platelet-rich plasma for open meniscal repair in young patients: any benefit? Knee Surg Sports Traumatol Arthrosc 2015; 23: 51-58.

19. Griffin J W, Hadeed M M, Werner B C, Diduch D R, Carson E W, Miller M D. Platelet-rich plasma in meniscal repair: does augmentation improve surgical outcomes? Clin Orthop Relat Res 2015; 473: 1665-1672.

20. Shin K H, Lee H, Kang S, Ko Y J, Lee S Y, Park J H, et al. Effect of leukocyte-rich and platelet-rich plasma on healing of a horizontal medial meniscus tear in a rabbit model. Biomed Res Int, 2015.

21. Maher S A, Rodeo S A, Doty S B, Brophy R, Potter H, Foo L F, et al. Evaluation of a porous polyurethane scaffold in a partial meniscal defect ovine model. Arthroscopy 2010; 26: 1510-1519.

22. Arnoczky S P, Cook J L, Carter T, Turner A S. Translational models for studying meniscal repair and replacement: what they can and cannot tell us. Tissue Eng Part B Rev 2010; 16: 31-39.

23. Chevrier A, Nelea M, Hurtig M B, Hoemann C D, Buschmann M D. Meniscus structure in human, sheep, and rabbit for animal models of meniscus repair. J Orthop Res 2009; 27: 1197-1203.

24. Chiari C, Koll e, U, Dorotka R, Eder C, Plasenzotti R, Lang S, et al. A tissue engineering approach to meniscus regeneration in a sheep model. Osteoarthritis Cartilage 2006; 14: 1056-1065.

25. Brophy R H, Cottrell J, Rodeo S A, Wright T M, Warren R F, Maher S A. Implantation of a synthetic meniscal scaffold improves joint contact mechanics in a partial meniscectomy cadaver model. J Biomed Mater Res A 2010; 92: 1154-1161.

26. Oda S, Otsuki S, Kurokawa Y, Hoshiyama Y, Nakajima M, Neo M. A new method for meniscus repair using type I collagen scaffold and infrapatellar fat pad. J Biomater Appl 2015.

27. Wardale J, Mullen L, Howard D, Ghose S, Rushton N. An ex vivo model using human osteoarthritic cartilage demonstrates the release of bioactive insulin-like growth factor-1 from a collagen-glycosaminoglycan scaffold. Cell Biochem Funct 2015; 33: $277-284$.

28. Smith E L, Kanczler J M, Gothard D, Roberts C A, Wells J A, White L J, et al. Evaluation of skeletal tissue repair, part 1: assessment of novel growth-factor-releasing hydrogels in an ex vivo chick femur defect model. Acta Biomaterialia 2014; 10: 4186-4196.

29. Barry F P. Biology and clinical applications of mesenchymal stem cells. Birth Defects Res C Embryo Today 2003; 69: 250-256.

30. Yuan X, Eng G M, Arkonac D E, Chao P H, Vunjak-Novakovic G. Endothelial cells enhance the migration of bovine meniscus cells. Arthritis Rheumatol 2015; 67: 182-192.

31. Yuan X, Arkonac D E, Chao P H, Vunjak-Novakovic G. Electrical stimulation enhances cell migration and integrative repair in the meniscus. Sci Rep 2014; 14: 3674.

32. Mauck R L, Martinez-Diaz G J, Yuan X, Tuan R S. Regional multilineage differentiation potential of meniscal fibrochondrocytes: implications for meniscus repair. Anat Rec 2007; 290: 48-58.

33. Webber R J, York J L, Vanderschildren J L, Hough A J. An organ culture model for assaying wound repair of the fibrocartilagenous knee joint meniscus. Am J Sports Med 1989; 17: 393-400.

34. Gonzales V K, de Mulder E L, de Boer T, Hannink G, van Tienen T G, van Heerde W L, et al. Platelet-rich plasma can replace foetal bovine serum in human meniscal cell cultures. Tiss Eng Part C Methods 2013; 19: 892-899.

35. Chung B M, Rotty J D, Coulombe P A. Networking galore: intermediate filaments and cell migration. Curr Opin Cell Biol 2013; 25: 600-612.

36. Hinz B, Celetta G, Toamsek J J, Gabbiani G, Chaponnier C. Alpha-smooth muscle actin expression upregulates fibroblast contractile activity. Mol Biol Cell 2001; 12: 2730-2741.

37. Kinner B, Spector M. Smooth muscle actin expression by human articular chondrocytes and their contraction of a collagen-glycosaminoglycan matrix in vitro. J Orthop Res 2001; 19: 233-241.

38. Mueller S M, Schneider T O, Shortkroff S, Breinan H A, Spector M. Alpha-smooth muscle actin and contractile behaviour of bovine meniscus cells seeded in type I and type II collagen-GAG matrices. J Biomed Mater Res 1999; 45: 157-166.

39. Povysil C, Dundr P, Tvrdík D, Podskubka A, Kana R, Horák M. Desmin-positive and alpha-smooth muscle actin positive chondrocytes in human defective articular cartilage--preliminary report. Cesk Patol 2005; 41: 133-136.

40. Liu C Y, Kao W W. Lumican promotes corneal epithelial wound healing. Methods Mol Biol 2012; 836: 285-290.

41. Liu X J, Kong F Z, Wang Y H, Zheng J H, Wan W D, Deng C L, et al. Lumican Accelerates Wound Healing by Enhancing _2_1 Integrin-Mediated Fibroblast Contractility. PLoS One 2013; 8: e67124.

42. Radwanska A, Litwin M, Lowak D, Baczynska D, Wegrowski Y, Maquart $\mathrm{F} \mathrm{X}$, et al. Overexpression of lumican affects the migration of human colon cancer cells through up-regulation of gelsolin and filamentous actin reorganization. Exp Cell Res 2012; 318: 2312-2323.

43. Roubelakis M G, Trohatou O, Roubelakis A, Mill E, Kalaitzopoulos I, Papazoglou G, et al. Platelet rich plasma (PRP) promotes fetal mesenchymal stem/stromal cell migration and would healing process. Stem Cell Rev 2014; 10: 417-428.

44. Cacares M, Hidalgo R, Sanz A, Martinez J, Riera P, Smith P C. Effect of platelet-rich plasma on cell adhesion, cell migration and myofibroblastic differentiation in human gingival fibroblasts. J Periodontol 2008; 79: 714-720.

45. Kushida S, Kakudo N, Suzuki K, Kusumoto K. Effects of platelet-rich plasma on proliferation and myofibroblastic differentiation in human dermal fibroblasts. Ann Plast Surg 2013; 71: $219-224$.

46. Batson J, Astin J W, Nobes C D. Regulation of contact inhibition of locomotion by Eph-signalling. J Microsc 2013; 251: 232-241. 